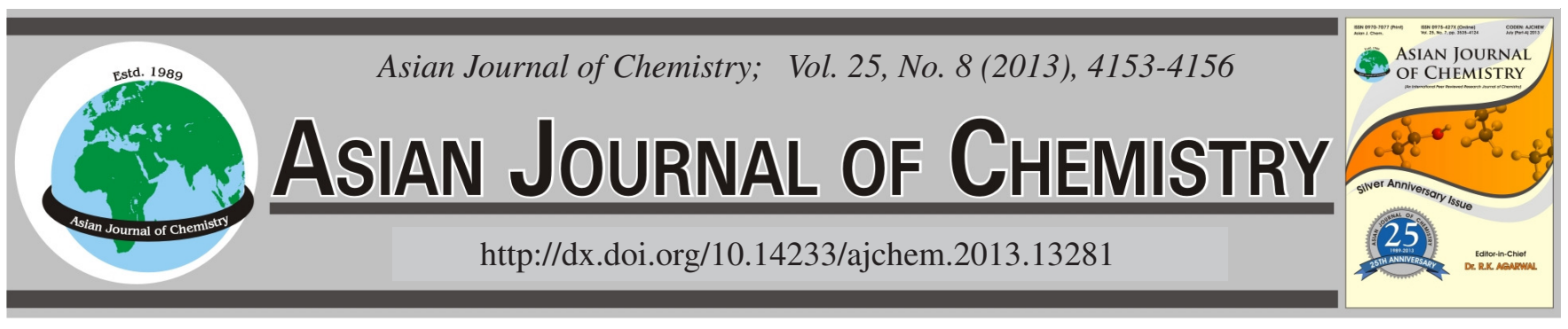

\title{
Various Elements of Heat Sources within an Optimized Photonic Crystal Vertical Cavity Surface Emitting Laser: Influence of Hole Etching Depth
}

\section{SAEID MARJANi}

Young Researchers Club, Arak Branch, Islamic Azad University, Arak, Iran

Corresponding author: E-mail: saeidmarjani@yahoo.com

\section{INTRODUCTION}

A crystal is a periodic arrangement of atoms or molecules. The pattern which the atoms or molecules are repeated in space is the crystal lattice. The crystal presents a periodical potential to an electron propagating through it and both the constituents of the crystal and the geometry of the lattice dictate the conduction properties of the crystal. If the dielectric constants of the materials in the crystal are sufficiently different and if the absorption of light by the materials is minimum, then the refractions and reflections of light from all the different interfaces can present many of the same phenomena for photons that the atomic potential produces for electrons.

Fig. 1 is a schematic representation of several threedimensional lattices of spheres in a cubic cell. The simplest lattice is formed by the blue spheres at the corners of the cube. If we add the dark red spheres at the centers of the faces, we obtain a face-centered cubic (or fcc) lattice. The fcc lattice vectors are $\left({ }^{\wedge} x+{ }^{\wedge} y\right) a / 2,\left({ }^{\wedge} y+{ }^{\wedge} z\right) a / 2$ and $\left({ }^{\wedge} x+{ }^{\wedge} z\right) a / 2$. Finally, if we add the pink spheres, which represent another fcc lattice that is shifted by $(\mathrm{a} / 4, \mathrm{a} / 4, \mathrm{a} / 4)$ relative to the blue spheres, then we obtain a diamond lattice ${ }^{1}$.

Vertical cavity surface emitting laser is one of the key light sources used in high performance optical communication systems where single mode operation, high-output power, high speed modulation and low manufacturing cost are necessary ${ }^{2}$. High optical gain in the active area and high thermal conductivity

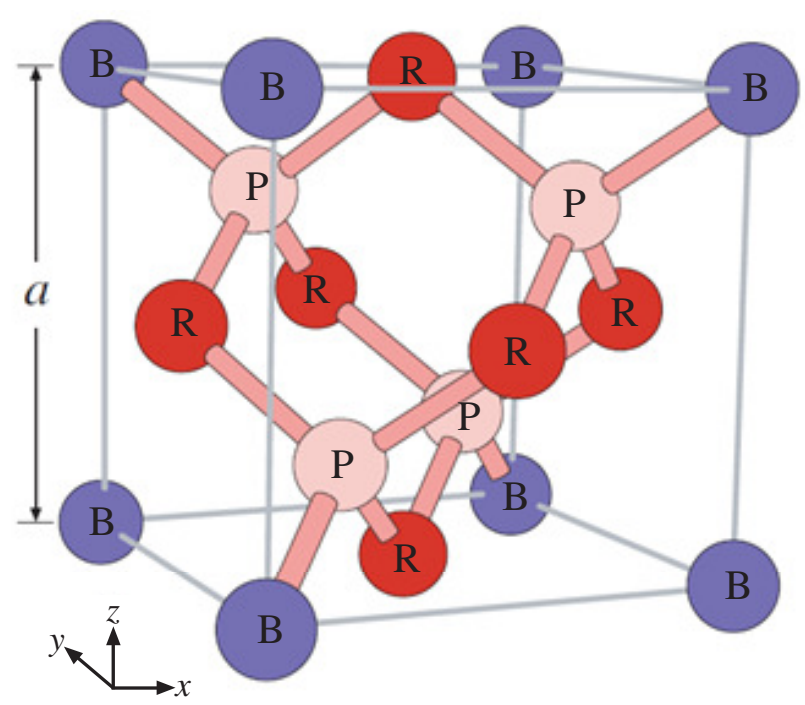

Fig. 1. Ball-and-stick (atomic) representation of several three-dimensional lattices in a cubic supercell, with a lattice constant a. Blue balls (B) alone form a simple cubic lattice. Adding the red balls (R) produces a face-centered cubic (fcc) lattice. Adding the pink balls (P) as well produces a diamond lattice, with stick bonds (four bonds per ball)

in the reflecting mirrors are the main difficulties in developing vertical cavity surface emitting lasers which are used in the field of optical spectroscopy ${ }^{3}$. Recently, we proposed a structure for decreasing the threshold current of vertical cavity surface 
emitting lasers and showed proposed structure decreases the threshold current about $76.52 \%$ from $2.3 \mathrm{~mA}$ to $0.6 \mathrm{~mA}^{4}$. The goal of this paper is to introduce the influence of the hole etching depth on various elements of heat sources within the active region of a photonic crystal vertical cavity surface emitting laser (VCSEL) at a constant bias power by combining of structures presented by Marjani et al. ${ }^{4,5}$.

\section{THEORY AND MODEL}

In VCSEL modeling, we must consider the electrical, optical and thermal interaction during laser performance. Thus the base of simulation is to solve Poisson and continuity equations for electrons and holes ${ }^{6}$. Poisson's equation is defined by ${ }^{6}$ :

$$
\nabla \cdot(\varepsilon \nabla \Psi)=\rho
$$

where $\Psi$ is electrostatic potential, $\rho$ is local charge density and $\varepsilon$ is local permittivity. The continuity equations of electron and hole are given by Piperak ${ }^{7}$ :

$$
\begin{aligned}
& \frac{\mathrm{dn}}{\mathrm{dt}}=\mathrm{G}_{\mathrm{n}}-\mathrm{R}_{\mathrm{n}}+\frac{1}{\mathrm{q}} \nabla \cdot \mathrm{j}_{\mathrm{n}} \\
& \frac{\mathrm{dp}}{\mathrm{dt}}=\mathrm{G}_{\mathrm{p}}-\mathrm{R}_{\mathrm{p}}+\frac{1}{\mathrm{q}} \nabla \cdot \mathrm{j}_{\mathrm{p}}
\end{aligned}
$$

where $\mathrm{n}$ and $\mathrm{p}$ are the electron and hole concentration, $\mathrm{J}_{\mathrm{n}}$ and $J_{p}$ are the electron and hole current densities, $G_{n}$ and $G_{p}$ are the generation rates for electrons and holes, $R_{n}$ and $R_{p}$ are the recombination rates and $\mathrm{q}$ is the magnitude of electron charge.

The fundamental semiconductor eqns. 1-3 are solved selfconsistently together with Helmholtz and the photon rate equations. The applied technique for solution of Helmholtz equation is based on improved effective index model ${ }^{8}$ which shows accuracy for great portion of preliminary problems. This model is very good adapted to simulation of laser structures and it is often called effective frequency method ${ }^{9}$.

Two-dimensional Helmholtz equation is solved to determine the transverse optical field profile and it is given by following eqn. ${ }^{6}$ :

$$
\nabla^{2} \mathrm{E}(\mathrm{r}, \mathrm{z}, \varphi)+\frac{\omega_{0}}{\mathrm{c}^{2}} \varepsilon(\mathrm{r}, \mathrm{z}, \varphi, \omega) \mathrm{E}(\mathrm{r}, \mathrm{z}, \varphi)=0
$$

where $\omega$ is the frequency, $\varepsilon(\mathrm{r}, \mathrm{z}, \varphi, \omega)$ is the complex dielectric permittivity, $\mathrm{E}(\mathrm{r}, \mathrm{z}, \varphi)$ is the optical electric field and $\mathrm{c}$ is the speed of light in vacuum. The light power equation relates electrical and optical models. The photon rate equation is given by following eqn. ${ }^{6}$ :

$$
\frac{\mathrm{dS}_{\mathrm{m}}}{\mathrm{dt}}=\left(\frac{\mathrm{c}}{\mathrm{N}_{\mathrm{eff}}} \mathrm{G}_{\mathrm{m}}-\frac{1}{\tau_{\mathrm{ph}_{\mathrm{m}}}}-\frac{\mathrm{cL}}{\mathrm{N}_{\mathrm{eff}}}\right) \mathrm{S}_{\mathrm{m}}+\mathrm{R}_{\mathrm{sp}_{\mathrm{m}}}
$$

where $S_{m}$ is the photon number, $G_{m}$ is the modal gain, $R_{s p_{m}}$ is the modal spontaneous emission rate, $\mathrm{L}$ represents the losses in the laser, $\mathrm{N}_{\text {eff }}$ is the group effective refractive index, $\tau_{\mathrm{ph}}$ is the modal photon lifetime and $\mathrm{c}$ is the speed of light in vacuum. The heat flow equation has the form ${ }^{6}$ :

$$
\mathrm{C} \frac{\partial \mathrm{T}_{\mathrm{L}}}{\partial \mathrm{t}}=\nabla\left(\kappa \nabla \mathrm{T}_{\mathrm{L}}\right)+\mathrm{H}
$$

where $\mathrm{C}$ is the heat capacitance per unit volume, $\kappa$ is the thermal conductivity, $\mathrm{H}$ is the generation, $\mathrm{T}_{\mathrm{L}}$ is the local lattice temperature and $\mathrm{H}$ is the heat generation term.
The heat generation equation has the form ${ }^{6}$ :

$\mathrm{H}=\left(\frac{\mid \overrightarrow{\left.J_{n}\right|^{2}}}{q \mu_{n} n}+\frac{\overrightarrow{\left.J_{p}\right|^{2}}}{q \mu_{p} n}\right)+\begin{aligned} & q(R-G)\left[\phi_{p}-\phi_{n}+T_{L}\left(P_{p}-P_{n}\right)\right]- \\ & T_{L}\left(\overrightarrow{J_{n}} \nabla P_{n}+\vec{J}_{p} \nabla P_{p}\right)\end{aligned}$

where:

$\left(\frac{\left|\mathrm{J}_{\mathrm{n}}\right|^{2}}{\mathrm{q} \mu_{\mathrm{n}} \mathrm{n}}+\frac{\overrightarrow{\left.\mathrm{J}_{\mathrm{p}}\right|^{2}}}{\mathrm{q} \mu_{\mathrm{p}} \mathrm{n}}\right)$ is the Joule heating term,

$\mathrm{q}(\mathrm{R}-\mathrm{G})\left[\phi_{\mathrm{p}}-\phi_{\mathrm{n}}+\mathrm{T}_{\mathrm{L}}\left(\mathrm{P}_{\mathrm{p}}-\mathrm{P}_{\mathrm{n}}\right)\right]$ is the recombination and generation heating and cooling term, $-\mathrm{T}_{\mathrm{L}}\left(\overrightarrow{\mathrm{J}_{n}} \nabla \mathrm{P}_{\mathrm{n}}+\overrightarrow{\mathrm{J}_{\mathrm{p}}} \nabla \mathrm{P}_{\mathrm{p}}\right)$ accounts for the Peltier and Thomson effects.

Eqns. 1-7 provide an approach that can account for the mutual dependence of electrical, thermal, optical and elements of heat sources.

Fig. 2(a) shows the schematic structure of photonic crystal VCSEL device, which is used for simulation. The VCSEL device consists of an active region consists of six quantum wells where the well is $5.5 \mathrm{~nm} \operatorname{In}_{0.76} \mathrm{Ga}_{0.24} \mathrm{As}_{0.82} \mathrm{P}_{0.18}$ and the barrier is $8 \mathrm{~nm} \operatorname{In}_{0.48} \mathrm{Ga}_{0.52} \mathrm{As}_{0.82} \mathrm{P}_{0.18}$. In both sides of this active region, there is $\mathrm{InP}$ and on top of it GaAs. 30 layers of the top mirror are made of $\mathrm{GaAs} / \mathrm{Al}_{0.33} \mathrm{Ga}_{0.67} \mathrm{As}$ with index of refraction of layers 3.38 and 3.05 respectively and 28 layers of the bottom mirror are made of GaAs/AlAs with index of refraction of layers 3.38 and 2.89, respectively. The incorporation of a high aluminum content layer in two DBR periods above the active region allows for selective oxidation ${ }^{10}$. In this work, the etching depth was continued down to the top of lower InP layer. Triangular-lattice air holes are formed in the upper pairs of top DBR. The optical confinement is achieved by means of seven air holes where the center is missed off to make the defect region, as shown in Fig. 2(b). The crucial photonic crystal parameters are connected by the hole etch depth (dE), the air hole diameter (a), the pitch (b) and the optical aperture diameter (r), are defined in Fig. 2(b).

\section{RESULTS AND DISCUSSION}

In the present work, the hole etching depth was varied between 2 until $6 \mu \mathrm{m}$ at a constant bias power and its influences on the various elements of heat sources of the device was analyzed and discussed. Fundamentally, the heat power peaks at the MQW region for all heat source elements. The highest contributor of heat power comes from the Peltier-Thomson heat power (up to $2.7522 \mathrm{e} 10 \mathrm{~W} / \mathrm{cm}^{3}$ ) followed by the recombination heat power $\left(5.1179 \mathrm{e} 8 \mathrm{~W} / \mathrm{cm}^{3}\right)$ and finally, the joule heat power (up to $6.5408 \mathrm{e} 7 \mathrm{~W} / \mathrm{cm}^{3}$ ).

Fig. 3(a) shows the joule heat power within a vertical cross-section of the active region at a bias power of $36 \mathrm{~mW}$. As can be seen from Fig. 3(a), increasing of the hole etching depth causes the reduction of the joule heat power which should be mainly due to lower current density in Pin $=36 \mathrm{~mW}$. By increasing of the depth into the active region, the peak of joule heat power increases.

Fig. 3(b) shows the Peltier-Thomson heat power within a vertical cross-section of the active region while the hole etching depth is changed. As shown in the Fig. 3(b), when the hole 


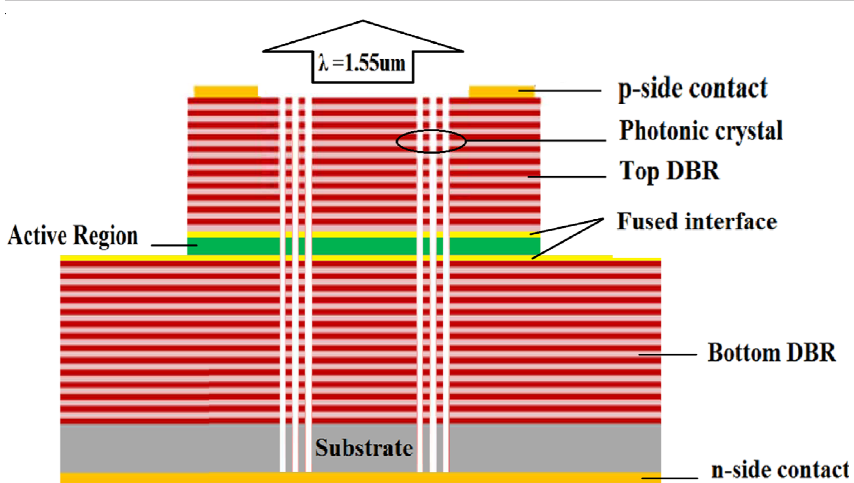

(a)

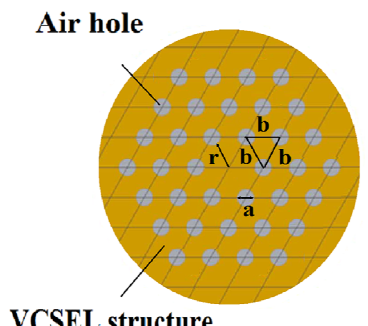

(b)

Fig. 2. (a) Schematic structure of the PhC VCSEL device and (b) Top view of the triangular-lattice air holes pattern

etching depth increases, the Peltier-Thomson heat power shows the same rule as that at the joule heat power which should be mainly due to lower current density. Peltier-Thomson heat is transferred between carriers and lattice as current flows along a gradient of the thermoelectrical power and it changes with the density of states, carrier concentration and temperature. When electrons enter a material with under conduction band edge, they suddenly display extra kinetic energy (hot electrons) that is finally scattered to the lattice. Vice versa, electrons need to receive additional energy from the lattice to leave the quantum well. For that reason, Thomson heat can be positive or negative.

Fig. 3(c) shows the recombination heat power within a vertical cross-section of the active region while the hole etching depth is changed. It can be found that the increment of the hole etching depth from 2 to $6 \mu \mathrm{m}$, leads to the upper recombination heat power at a bias power of $36 \mathrm{~mW}$, which should be mainly due to upper net recombination rate, including thermal generation of carriers.

\section{Conclusion}

In this work, we present the influence of the hole etching depth on various elements of heat sources within the active region of an optimized long wavelength $\mathrm{InGaAsP}$ photonic crystal oxide VCSEL at a constant bias power. The results indicate that increasing of the hole etching depth, decreases the joule and Peltier-Thomson heat powers but increases the recombination heat power into MQW region at a bias power of $36 \mathrm{~mW}$. Simulation results show that the highest contributor of heat power comes from the Peltier-Thomson heat power (up to $2.7522 \mathrm{e} 10 \mathrm{~W} / \mathrm{cm}^{3}$ ) followed by the recombination heat power $\left(5.1179 \mathrm{e} 8 \mathrm{~W} / \mathrm{cm}^{3}\right)$ and finally, the joule heat power (up to $\left.6.5408 \mathrm{e} 7 \mathrm{~W} / \mathrm{cm}^{3}\right)$.

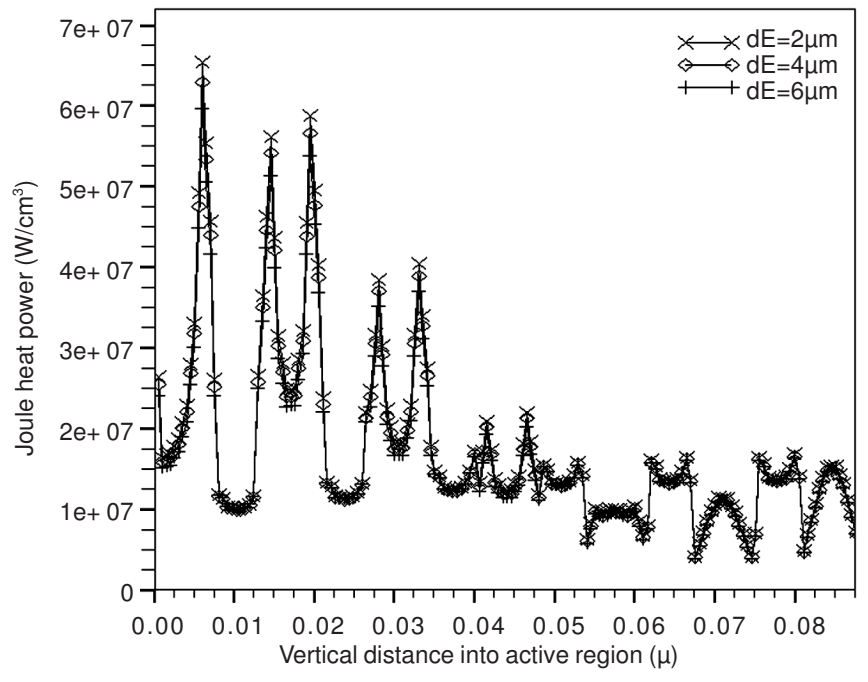

(a)

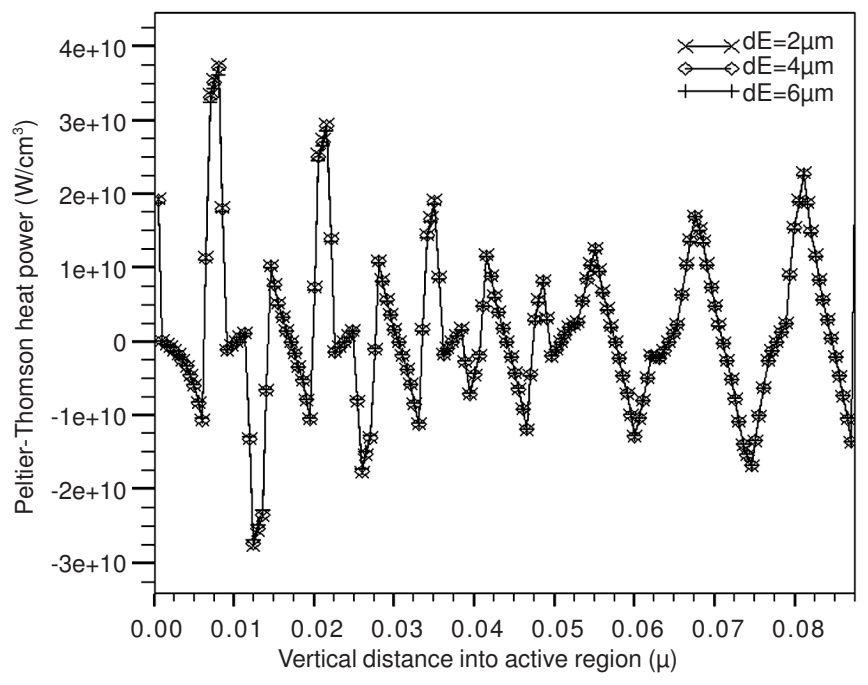

(b)

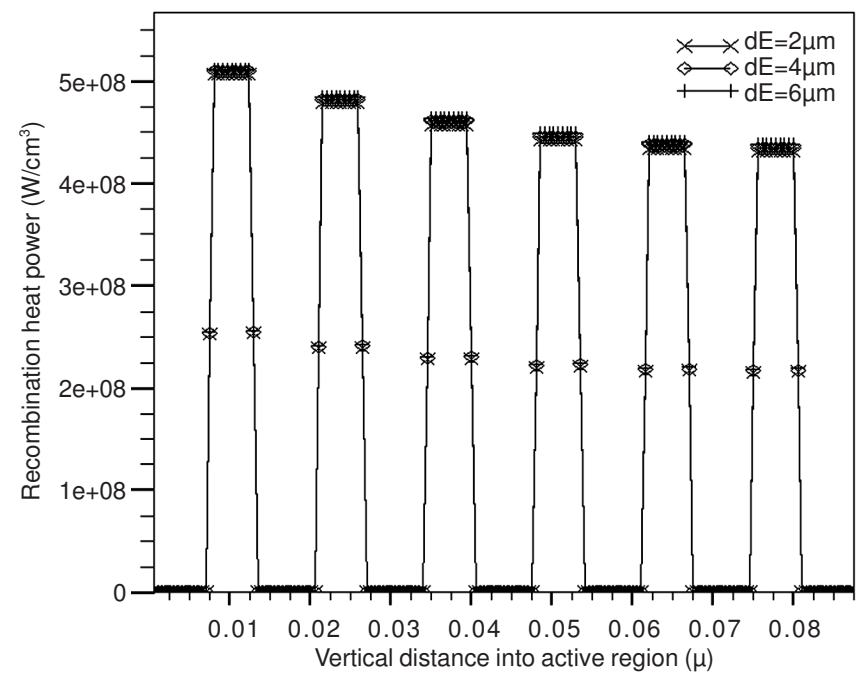

(c)

Fig. 3. Influence of the hole etching depth on various elements of heat sources within the active region (a) Joule heat power, (b) PeltierThomson heat power and (c) Recombination heat power 


\section{REFERENCES}

1. J.D. Joannopoulos, S.G. Johnson, R.D. Meade and J.N. Winn, Photonic Crystals: Molding the Flow of Light, Princeton, Univ. Press, edn. 2, pp. 94-99 (2008)

2. M. Dems, R. Kotynski and K. Panajotov, J. Opt. Express, 13, 3196 (2005).

3. E. Kapon and A. Sirbu, J. Nature Photon., 3, 27 (2009).

4. R. Faez, A. Marjani and S. Marjani, J. IEICE Electron. Express, 8, 1096 (2011)

5. S. Marjani and H. Marjani, Asian J. Chem., 24, 3194 (2012).
6. SILVACO International, ATLAS User's Manual, USA, SILVACO International Incorporated (2010)

7. J. Piprek, Semiconductor Optoelectronic Devices: Introduction to Physics and Simulation, Ch. 3 Carrier Transport and Ch. 6 Heat Generation and Dissipation UCSB: Academic Press, pp. 49-50 and 141-147 (2003).

8. G.R. Hadley, J. Opt. Lett., 20, 1483 (1995).

9. H. Wenzel and H.J. Wunsche, IEEE J. Quantum Electron., 33, 1156 (1997).

10. K.D. Choquette, K.M. Geib, C.I. Ashby, R.D. Twesten, O. Blum, H.Q. Hou, D.M. Follstaedt, B.E. Hammons, D. Mathes and R. Hull, IEEE J. Sel. Topics Quantum Electron., 3, 916 (1997). 